\title{
Concepto de Lugar Geométrico. Génesis de Utilización Personal y Profesional con Distintas Herramientas
}

\author{
The Concept of Locus. Genesis of Personal and Professional Use with \\ Different Tools
}

\begin{abstract}
Resumen
La finalidad de este artículo es precisar algunos de los elementos que organizan un espacio de trabajo efectivo para problemas de lugares geométricos en entornos tecnológicos. Se explora como 52 futuros profesores de matemáticas progresan en su concepción de lugares geométricos a través de la apropiación de las funcionalidades específicas de cada entorno (herramienta), en relación con su propia práctica como estudiantes y su futuro ejercicio profesional. Con base en sistemas de geometría dinámica se comparan tres herramientas, las diferentes representaciones matemáticas de los lugares geométricos generadas por ellas, tanto desde la perspectiva de su dinámica matemática como de sus funcionalidades didácticas. Las funcionalidades didácticas proporcionadas desde el diseñador se han estudiado desde el modelo Espacio de Trabajo Matemático (ETM). Este modelo pone de relieve la necesidad de articular para el trabajo geométrico los niveles epistemológico y cognitivo a través de diferentes génesis de razonamiento (visual-discursiva, instrumental y discursiva).
\end{abstract}

Palabras clave: Lugares Geométricos. Geometría Dinámica. Génesis Instrumental. Razonamiento Geométrico. Espacio de Trabajo Matemático.

\begin{abstract}
The purpose of this article is to clarify some of the elements involved in an effective workspace for problems related to geometric loci in technological environments. It analyzes how 52 prospective math teachers progress in their conception of the concept of geometric locus through the appropriation of the specific features of each environment (tool) in relation to their own practice as students and their future professional activity. The mathematical representations of loci generated by three different dynamic geometry tools have been compared,
\end{abstract}

\footnotetext{
* Doctora, Universidad Complutense de Madrid (UCM), Instituto de Matemática Interdisciplinar (IMI), Madrid, España. Dirección postal: Facultad de Ciencias Matemáticas, Ciudad Universitaria, Plaza de Ciencias 3, 28040 Madrid, España. E-mail: igomezchacon@mat.ucm.es

** Doctor, Universidad de Vigo (UVigo), Vigo, España. Dirección postal: Department of Applied Mathematics I, Escola de Enxeñería Forestal, University of Vigo at Pontevedra, Campus A Xunqueira, 36005 Pontevedra, Spain. E-mail: fbotana@uvigo.es

Doctor, Instituto de Matemática Interdisciplinar (IMI) y Universidad Complutense de Madrid (UCM), Madrid, España. Dirección postal: Facultad de Ciencias Matemáticas, Ciudad Universitaria, Plaza de Ciencias 3, 28040 Madrid, España. E-mail: jesusesc@ucm.es

Doctor, Universidad Rey Juan Carlos (URJC), Madrid, España. Dirección postal: Calle Tulipán s/n. 28933 Móstoles (Madrid). E-mail: miguelangel.abanades@urjc.es
} 
both from the perspective of their mathematical dynamics and their didactic functions. The didactic functionalities provided from the designer have been studied from the Mathematical Working Space model (MWS). This model highlights the need to coordinate the geometric work on the epistemological and cognitive levels through different genesis of reasoning (visual-discursive, instrumental and discursive).

Keywords: Geometric Loci. Dynamic Geometry. Instrumental Genesis. Geometric Reasoning. Mathematical Working Space.

\section{Resumo}

A finalidade de este artigo é o de explicitar de forma exacta e completa alguns dos elementos que organizam um espaço de trabalho efectivo para problemas de lugares geométricos em ambientes tecnológicos. Explora-se como 52 futuros professores de matemática progridem na sua concepção de lugares geométricos através da apropriação das funcionalidades de cada ambiente (ferramenta), em relação com a própria prática como estudiantes e o seu futuro exercício profissional. Com base em sistemas de geometria dinâmica comparam-se três ferramentas, as diferentes representações matemáticas dos lugares geométricos geradas por elas, tanto da perspectiva da sua dinâmica matemática como das suas funcionalidades didácticas. As funcionalidades didácticas proporcionadas pelo desenhador estudaram-se desde o modelo Espaço de Trabalho Matemático (ETM). Este modelo põe em relevo a necessidade de articular para o trabalho geométrico os níveis epistemológicos e cognitivos através de diferentes géneses de raciocínio (visual, instrumental e discursiva).

Palavras-chave: Lugares Geométricos. Geometria Dinâmica. Gênese Instrumental. Raciocínio Geométrico. Espaço de Trabalho Matemático.

\section{Introducción}

Según Balacheff y Kaput (1996), el principal impacto de las nuevas tecnologías en los sistemas educativos es epistemológico y cognitivo, ya que ha contribuido a la producción de una nueva forma de realismo en los objetos matemáticos. El diseño del software desempeña una dimensión crucial en la consideración de este impacto. En este trabajo, examinamos y evaluamos las diferentes representaciones matemáticas de los lugares geométricos desarrolladas por tres herramientas desde la perspectiva de su dinámica matemática y sus funcionalidades didácticas. En estas formas de representación, el paso del mundo numérico al mundo algebraico no está exento de problemas y se requiere una comprensión mayor del comportamiento matemático de la continuidad y de los registros algebraicos.

El concepto de lugar es tan antiguo como las matemáticas o, en particular, como la geometría. De hecho, la humanidad siempre ha estado fascinada por las curvas, incluso antes de que se vieran como objetos matemáticos como se puede constatar en varias inscripciones prehistóricas. Los griegos comenzaron el estudio de curvas diferentes, desde los más simples (líneas, círculos, cónicas) de las curvas más complejas, obtenidos mediante el estudio de las trayectorias de los puntos construidos a partir de simples curvas. Es lo que llamamos hoy en día Lugares (loci). 
Con el desarrollo de las matemáticas, los matemáticos estudian curvas cada vez más complejas, de las que surgen problemas complicados, a menudo definidas en términos de Loci (Conchoide de Nicomedes, deltoides de Steiner). Además, en el siglo XIX, muchos mecanismos, desarrollados en Mecánica, se pueden definir en términos de Loci. Esto nos permite conocer el camino real de las partes de estos mecanismos, cuando, en principio, sólo eran conocidos los enfoques parciales (BOTANA; ABÁNADES, 2014).

Para situar el estudio parece pertinente entrar en el punto de vista conceptual geométrico de la definición de Locus con objeto de comprender aspectos epistemológicos que afecten a la dimensión cognitiva-instrumental del usuario cuando se utilizan las tres herramientas que se discuten en este artículo.

En los libros de texto la noción de Locus se introduce vinculada a construcciones con regla y compás, donde su aspecto constructivo y mecánico es claro en el contexto de las aplicaciones (caso1). Pero también, podemos encontrar la noción Locus en el contexto de las transformaciones, mostrando que estas transformaciones son herramientas muy eficaces para resolver problemas de Locus (caso 2), cuyo significado no es el mismo.

En el primer caso la definición vendría dada comoDefinición 1: Un lugar geométrico es el conjunto de puntos que satisfacen una determinada propiedad expresable a partir de una construcción geométrica realizada con regla y compás. La expresión "con regla y compás" puede ser un poco difusa, pero denota una forma de construcción y dicha propiedad se enuncia habitualmente en términos de distancias a puntos, rectas o circunferencias fijas en el plano y/o en términos del valor de un ángulo. Esta se conoce como la aproximación "clásica".

También, podemos encontrar otro tipo de formulación en el contexto de las transformaciones, en este caso nos referimos a un conjunto de puntos que son imágenes de un conjunto de puntos, se define como la imagen de un objeto bajo una aplicación o transformación: Si llamamos $f$ de la función: $\mathrm{M} \rightarrow \mathrm{N}=f(\mathrm{M})$, buscar el lugar geométrico de $\mathrm{N}$ es buscar el conjunto de todos los puntos $f(\mathrm{M})$.O también, lo encontramos expresado como:Un lugar geométrico es el conjunto de todos puntos del plano que verifican una propiedad determinada. Por lo tanto:

Si L es un lugar geométrico definido por la propiedad P, se verifica que:

a) Todo punto de $L$ posee la propiedad $P$.

b) Todo punto que posee la propiedad $\mathrm{P}$ pertenece a $\mathrm{L}$.

La condición b) puede sustituirse por la siguiente:

c) Todo punto no perteneciente a $\mathrm{L}$ no posee la propiedad $\mathrm{P}$. 
Si un punto $\mathrm{Q}(x, y)$ en el plano cartesiano pertenece a un lugar geométrico L, la condición que debe cumplir dicho punto Q conduce a una ecuación entre las variables $x$ e $y$ que es la llamada ecuación del lugar geométrico L.

En este último caso, Locus se define de forma funcional, teóricamente tenemos que considerar que un punto $\mathrm{Q}$ variable que pertenece a una figura $\mathrm{F}$ considerado como un conjunto de puntos (una línea recta, un círculo, ...) corresponde a un punto Q', la imagen de Q por la aplicación $f$. Locus tiene un doble significado: por una parte legitima el cambio de una figura sintética (un punto global de ver) a una figura como un conjunto de puntos, y permite a recomponer la figura. Cuando analizamos los libros de texto también se observa que la noción de lugar se presenta a menudo - a nivel de Secundaria y Bachillerato - de una manera dinámica: "considera un punto $\mathrm{M}$ que se mueve en una línea fija (L), y otro punto $\mathrm{N}$ que está vinculado a M, se mueve también. El problema consiste en encontrar en qué línea fija (L1) el punto N se moverá. Esta línea (L1) es llamado Lugar Geométrico de N si el punto M se mueve en la línea L.

El Lugar Geométrico (o la transformación de la figura) se identifica con una trayectoria del punto $\mathrm{N}$ construido como imagen de un punto móvil $\mathrm{M}$ en la figura (L). Este punto de vista dinámico evita la utilización de cuantificadores y del lenguaje de la teoría de conjuntos. A pesar de que esta elección didáctica no corresponde a la noción de movimiento que se presenta en Física, sin embargo inspirados en el estudio histórico, nosotros hemos adoptado esta interpretación dinámica "punto móvil en una curva" como fase intermedia que puede ayudar al estudiante a comprender el sentido de transformación.

Esta distinción entre trayectoria y locus expresada en estas definiciones se refleja en el software de geometría dinámica mediante dos herramientas "LugarGeométrico", y "Traza". Utilizar la herramienta "LugarGeométrico" requiere de una aprehensión operatoria para hacer fecunda la intuición de la figura. La visualización del concepto no sólo icónica requiere de la toma de conciencia de las propiedades que están ligadas a las operaciones que se efectúan, bien para construir una figura o bien para transformarla (JANH, 2002; GÓMEZ-CHACÓN; ESCRIBANO, 2014; ABÁNADES et. al., 2015).

En este artículo nos limitaremos a los lugares geométricos planos que se pueden obtener con los entornos de geometría actuales, es decir, el conjunto de posiciones de un punto $\mathrm{T}$ dependiente de otro punto $\mathrm{M}$, ligado este a un camino lineal, en una cierta

Se ha utilizado la denominación entre comillas ya que se trata de la denominación exacta del comando en el software GeoGebra no se trata de un nuevo concepto. 
construcción con regla y compás. La estrategia habitual consiste en muestrear el camino de M y, para cada muestra, calcular la correspondiente posición de T. Estas posiciones se almacenan en una lista de pares de coordenadas numéricas, que es, en la mayoría de sistemas, el objeto que representa el lugar. Algunos entornos de geometría dinámica utilizan sencillas reglas para unir puntos contiguos en la lista y presentar así un resultado visualmente continuo. Sin embargo, esto puede producir resultados aberrantes debidos a discontinuidades o errores numéricos (BOTANA, 2002).

La finalidad de este artículo es precisar algunos de los elementos que organizan un espacio de trabajo efectivo para el estudio de lugares geométricos en entornos tecnológicos. Para ello se consideran tres herramientas (herramienta 1= "LugarGeométrico" en GeoGebra, herramienta 2= "EcuaciónLugar" en GeoGebra, herramienta 3= Prototipo "LocusGC") que se analizarán desde el punto de vista de las representaciones matemáticas que se generan. Se explorará como 52 graduados en matemáticas y futuros profesores de Secundaria progresan en su concepción de lugares geométricos a través de la apropiación de las funcionalidades específicas de cada herramienta, en relación con su propia práctica como estudiantes y su futuro ejercicio profesional.

Los expertos (desarrolladores) del Prototipo "LocusGC", que participan en esta investigación, plantean el enriquecimiento de los sistemas de geometría dinámica mediante sistemas de Álgebra computacional. Para ello se centran en la mejora de los principales problemas constatados en los sistemas de geometría dinámica: continuidad, cálculo de lugares geométricos, demostración y descubrimiento (BOTANA, 2007). La herramienta implementada en el prototipo LocusGC ha sido incorporada a GeoGebra, en su versión instalable de escritorio, con posterioridad al estudio recogido en este trabajo.

Desde la perspectiva del desarrollador, el problema de la continuidad no se considera sólo como una implementación correcta en la herramienta de estrategias de visualización, sino que conlleva un comportamiento matemático al que prestar atención en la formación del profesorado.

\section{Comportamiento matemático e instrumental de las herramientas}

Como hemos indicado la primera aproximación para el estudio de un problema de lugar geométrico es el uso de la traza. Dado un punto móvil, podemos indicar que GeoGebra dibuje el rastro que deja este punto al ser movido. La traza dejada por el punto móvil permite 
dar una idea intuitiva del lugar geométrico que representa, pero realmente es una aproximación muy grosera en la que además se pierde el dinamismo de la construcción. Esta estrategia de utilización de la traza se remonta a uno de los primeros interfaces gráficos, desarrollado hace ya medio siglo (SUTHERLAND, 2003).

\section{1 Herramienta "LugarGeométrico" en GeoGebra}

Para dibujar un lugar con una cierta precisión, es más adecuado utilizar una herramienta específica, "LugarGeométrico". A partir del punto que define el lugar y del punto que hace moverse a ese punto, GeoGebra realiza una aproximación numérica (pero sofisticada) del lugar geométrico. Esta construcción no tiene mayor estructura (es poco más que una "nube de puntos") pero se mantiene el dinamismo y hay una cierta precisión. La práctica totalidad de entornos geométricos actuales intenta sugerir la trayectoria uniendo posiciones sucesivas del punto trazador mediante heurísticos ad-hoc para estimar esta contigüidad. Y, aunque para construcciones normales, estas reglas son usualmente adecuadas y devuelven resultados apreciablemente correctos, es fácil encontrar situaciones donde el lugar mostrado exhibe anomalías. La Figura 1 muestra cómo GeoGebra produce una representación parcial y distorsionada del lugar al ampliar la escala de la construcción.

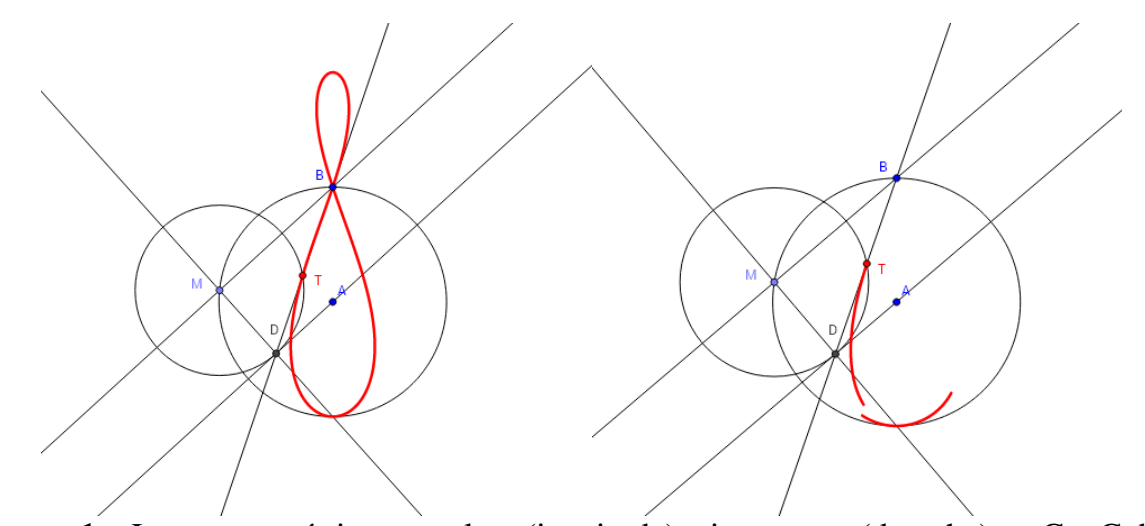

Figura 1 - Lugar geométrico completo (izquierda) e incorrecto (derecha) en GeoGebra.

Aunque el comando "LugarGeométrico" en GeoGebra admite diferentes tipos de argumentos $^{2}$, aquí usaremos el modelo que acepta dos argumentos, siendo el primero de ellos el punto cuya trayectoria describe el lugar y el segundo, necesariamente ligado a un objeto lineal, aquel punto cuyo movimiento genera el anterior. Obsérvese que el lugar obtenido es un objeto auxiliar para GeoGebra del que se ignora cualquier información algebraica. La

2 Ver http://wiki.geogebra.org/es/Comando_LugarGeom\%C3\%A9trico 
restricción impuesta acerca de las dos opciones admisibles por GeoGebra es a nuestro juicio irrelevante. Desechar la especificación de un deslizador como segundo argumento del comando no tiene repercusiones cognitivas. Cuestión aparte es la limitación impuesta a los lugares efectivamente computables. Considérese, por ejemplo, el lugar descrito por el punto medio de un segmento cuyos extremos reposan en sendos puntos móviles sobre dos circunferencias. Mientras que la activación de la traza del punto y el consecuente arrastre de los extremos del segmento sobre sendas circunferencias sugeriría incluso a un usuario inexperto que el lugar buscado no es lineal, sino bidimensional, el comando "LugarGeométrico" no es aplicable a este problema pues el punto del lugar buscado posee dos grados de libertad, es decir, depende de dos puntos sin relación alguna entre ellos. Así pues el diseñador de actividades que involucren la búsqueda de lugares geométricos ha de ser consciente de las limitaciones inherentes a la herramienta utilizada. Téngase en cuenta también que esta aproximación, limitada, como se ha dicho, a sencillas dependencias lineales, puede introducir incertidumbres cognitivas en el usuario: la mera observación de una curva imposibilita, en general, su categorización. Un ejemplo ilustrativo es el cálculo del lugar geométrico conocido como Caracol de Pascal, cuya sencilla construcción se detalla en el análisis del problema 2 en la sección 3.4 la curva obtenida (ver Figura3) es inclasificable a simple vista, al corresponder con una ecuación de grado 4. Sirvan estos ejemplos para ilustrar la complejidad de implementar procedimientos y herramientas como metáforas de procesos cognitivos y prácticas matemáticas (JACKIW, 2010).

\subsection{Herramienta "EcuaciónLugar" en GeoGebra}

En la versión actual de GeoGebra se incluye el comando "EcuaciónLugar" que permite calcular la ecuación de los lugares y la representa como una curva implícita. Básicamente, este comando considera las ecuaciones algebraicas de los elementos involucrados en el lugar y realiza un proceso de eliminación algebraica, basado en bases de Groebner, que devuelve una ecuación conteniendo únicamente las coordenadas simbólicas del punto que traza el lugar. Considerando el Caracol de Pascal, esta herramienta permite conocer la ecuación del lugar geométrico buscado. Pero, como es sabido, tal proceso de eliminación devuelve la menor variedad algebraica que contiene el lugar buscado (pudiendo pues incluir 
partes espurias, como se señala en la ayuda del comando "EcuaciónLugar"3). En el caso del Caracol de Pascal, GeoGebra devuelve no una curva algebraica de grado 4, sino una curva algebraica de grado 6 correspondiente al Caracol de Pascal con una circunferencia extra (ver Figura 3). Finalmente, esta herramienta es incapaz de realizar una discusión automática de las posibles degeneraciones o discutir la aparición de componentes algebraicas exóticas, lo que puede añadir incertidumbre en cuanto a la corrección del resultado.

\subsection{Prototipo "LocusGC" con GeoGebra}

La tercera alternativa que se usará solventa los problemas mencionados de inclusión de componentes espurias e indecidibilidad en caso de degeneración. Nótese que no resuelve todos los problemas surgidos al pasar de la herramienta "LugarGeométrico" a "EcuaciónLugar". Hay situaciones en las que los lugares geométricos buscados no son conjuntos algebraicos sino semialgebraicos, como una semicircunferencia, por ejemplo. El cálculo de este tipo de lugares implica el uso de técnicas de geometría real, actualmente en vías de implementación en GeoGebra.

Utilizando un reciente algoritmo de resolución de sistemas polinómicos con parámetros, GroebnerCover (MONTES; WIBMER, 2010), se ha construido el prototipo "LocusGC", en el que construcciones geométricas de lugares contenidas en un applet de GeoGebra son procesadas y la salida del algoritmo GroebnerCover es reintroducida en el applet, de forma que, para lugares estrictamente algebraicos, el resultado devuelto es matemáticamente correcto. Además, es una herramienta web que no necesita ningún tipo de instalación, sólo una conexión a internet.

\section{4 Interfaz de usuario y potencia expresiva de GeoGebra}

El manejo de "EcuaciónLugar" y de "LocusGC" es sencillo. Basta realizar una construcción geométrica en la que aparezca un lugar geométrico (utilizando la herramienta "LugarGeométrico"). Para utilizar "EcuaciónLugar", basta llamar a la instrucción desde la línea de comandos. En el caso de "LocusGC", basta con pulsar un botón.

Nótese también que otra limitación presente en los actuales interfaces consiste en la necesidad de construcción efectiva de un punto del lugar. La investigación de conjeturas

https://wiki.geogebra.org/es/Comando_Ecuaci\%C3\%B3nLugar 
geométricas plausibles está frecuentemente relacionada con el arrastre de algún objeto. Arzarello et al. (2002) definen, entre otras modalidades de arrastre, el dummy locus dragging como "...moving a basic point so that the drawing keeps a discovered property; the point which is moved follows a path, even if the users do not realize this: the locus is not visible and does not "speak" to the students, who do not always realize that they are dragging along a locus”. Un ejemplo canónico de esta situación es el circuncírculo de un triángulo como el lugar de los puntos cuyas proyecciones perpendiculares sobre los lados están alineadas. Si bien, tanto "EcuaciónLugar" como "LocusGC" podrían determinar ese lugar como una circunferencia, es la capacidad expresiva de GeoGebra (por otra parte equivalente a otros entornos estándar) la que limita la confirmación automática de esta conjetura.

\section{Estudio: contexto, marco interpretativo y metodología}

\subsection{Contexto y objetivo}

La experimentación se realiza con un grupo de 52 futuros profesores de matemáticas de Secundaria (estudiantes de un Máster Profesional en Educación Matemática Secundaria en la Universidad Complutense de Madrid).

La investigación sobre la enseñanza en contextos tecnológicos (LABORDE, 2001; LAGRANGE, 2013; GÓMEZ-CHACÓN; KUZNIAK, 2015) muestra que estos futuros profesores, que durante sus estudios de grado en Matemáticas han utilizado diferentes tipos de software, tienen escasos conocimientos sobre la enseñanza de las matemáticas y son poco conscientes del desarrollo de nociones matemáticas mediante el uso de software en una situación de enseñanza. Esto hace que sea necesario realizar estudios sobre el uso de software para enseñar en la formación docente.

En el presente trabajo se explora cómo este grupo de futuros profesores progresan en su concepción de lugares geométricos a través de la apropiación de las funcionalidades específicas de cada entorno (herramienta), en relación con su propia práctica como estudiantes y su futuro ejercicio profesional.

\subsection{Marco interpretativo y metodológico}


Dada una herramienta y una finalidad educativa es posible identificar las funcionalidades didácticas de la misma. Cerulli et al. (2005) señalan tres elementos en la definición de funcionalidad didáctica: (1) un conjunto de características de la herramienta, (2) un objetivo educativo y (3) las modalidades de empleo de la herramienta en un proceso de enseñanza / aprendizaje que se refiere a la finalidad educativa elegida.

Definir las modalidades de empleo de la herramienta depende del marco teórico de referencia que se considere (CERULLI et al., 2005). Aquí se utiliza el marco de Espacio de Trabajo Matemático (ETM) (KUZNIAK, 2011) como propuesta teórica y metodológica. Más específicamente, el modelo de Espacio de Trabajo Geométrico (ETG) y paradigmas. Este modelo destaca que en el dominio de la Geometría aparecen claramente tres paradigmas que se designan bajo los términos de Geometría I (o Geometría natural), Geometría II (o Geometría natural axiomática) y, finalmente, Geometría III (o Geometría axiomática formal). La idea que sustenta este modelo es que sólo se puede hablar de trabajo geométrico cuando la actividad del alumno es a la vez lo suficientemente coherente y compleja como para permitir la puesta en ejecución de una actividad de razonamiento. De esta manera, en cierto modo, hacen suyo el pensamiento de Gonseth (1945, p. 72): "Ser geómetra significa no confundir una evidencia nacida de la intuición con una información experimental, el resultado de una experiencia con la conclusión de un raciocinio". Estos autores introducen dos niveles conectados en la estructuración del ETG: el nivel epistemológico (la actividad geométrica en su dimensión puramente matemática) y el nivel cognitivo. Estos planos se articulan a través de tres génesis fundamentales: visual semiótica, instrumental, discursiva (Fig. 3), con objeto de garantizar un trabajo geométrico completo y coherente.

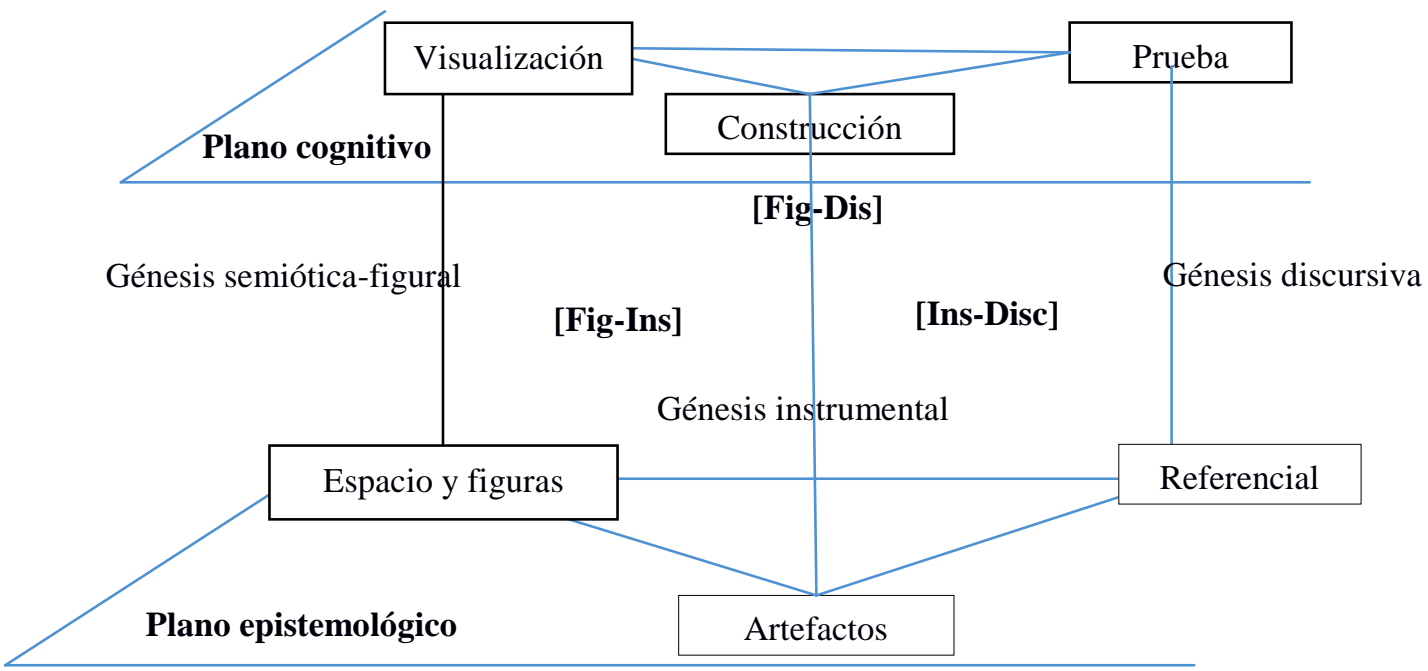

Figura 2 - Espacio de trabajo geométrico, génesis y planos en el ETM idóneo 
Siguiendo este modelo en el presente trabajo se observa y analiza la génesis instrumental de futuros profesores en problemas de lugares geométricos en un entorno de geometría dinámica (SGD) y la forma cómo esta génesis integra las herramientas en sus modalidades de empleo y funcionalidades clave en el aprendizaje matemático: intuición, exactitud, visualización-modelización algebraica. El acento está en los fenómenos instrumentales, el análisis de la interacción y la superposición entre el desarrollo de los conocimientos matemáticos (procesos de visualización y algebraicos) y la comprensión de los artefactos durante el experimento de enseñanza.

La aplicación de este modelo se utiliza como propuesta teórica y metodológica para en una primera fase:

- analizar las herramientas y problemática desde la perspectiva del experto y la concepción de trabajo matemático de los desarrolladores de las herramientas, ETM de referencia.

- analizar la diversidad de pensamientos matemáticos y cambios de registros en la resolución de los futuros profesores, ETM personal.

En una segunda fase, se reexaminan las producciones de los futuros profesores conjuntamente con los cuestionarios online (Anexo 1) para identificar:

- la percepción de los futuros profesores sobre la competencia de las herramientas desde el punto de vista de tipos de funcionamiento semióticofigural, instrumental y discursivo de cara a un uso profesional como futuros profesores.

La metodología utilizada ha sido multimétodos, combinando métodos cualitativos y cuantitativos. Como instrumentos de recogida de datos se les plantea la resolución de una serie de tres problemas de lugares geométricos a realizar con cada una de las tres herramientas informáticas (sec. 3.4). Los estudiantes deben realizar un protocolo detallado de la resolución de los mismos señalando procesos matemáticos, bloqueos y dificultades al resolver los problemas (Anexo 1). Se han realizado video-grabaciones de las sesiones de trabajo y entrevistas más detalladas a algunos alumnos.

Además, se les plantea para cada problema la realización de un cuestionario online de sus percepciones relativas a la competencia matemática de la herramienta y la comparación entre ellas (Anexo 2). En la valoración de las herramientas se tuvieron en cuenta distintos criterios de calidad de acuerdo con la finalidad del diseñador. Se plantearon dos niveles de valoración: un primer nivel, evaluación de la herramienta específica (solución/imagen 
esperada, exactitud-precisión matemática y satisfacción con el resultado matemático) y un segundo nivel comparativo (valorando las siguientes dimensiones: dimensión intuitiva, precisión matemática, sinergia entre visualización y registro algebraico). También cabe señalar que junto al análisis cualitativo se realizó un Análisis Estadístico Implicativo (SIA) (GRAS et al, 1997) buscando explorar la estructura de los ETM personales. Como indica Couturier (2007) este análisis estadístico permite establecer reglas de asociación en un conjunto de datos cruzando variables e individuos, marcando las tendencias de conjuntos de propiedades, usando una medida de carácter no lineal de tipo inferencial.

Las cuestiones abiertas concernientes a tipologías de resolución, tipologías de dificultad identificadas conscientemente y explicitadas por los sujetos, valoración de las herramientas, aspectos comparativos instrumentales-matemáticos y la caracterización de los sujetos fueron codificadas mediante un análisis cualitativo de contenido que permitía establecer categorías. Dos expertos realizaron estos análisis y cuantificaron sus frecuencias. De forma similar se compiló y codificó la matriz para el Análisis Implicativo para ser trabajada con el programa CHIC (COUTURIER, 2007) mediante variables binarias. Se analizará si los sujetos presentan una tendencia a cumplir la variable b cuando sabemos que cumplen la variable a. El objetivo principal del Análisis Implicativo es poner de relieve tales tendencias en un conjunto de propiedades. Este análisis nos permite abordar dos puntos de vista complementarios de la implicación entre las variables: una perspectiva global que busca cuantificar la calidad de cada una de las particiones asociadas con cada nivel de jerarquía, y un punto de vista local que se centra en la calidad de las reglas -asimilables a las clasesconstruidas en cada nivel.

Por último señalar que no se pretende establecer conclusiones para el conjunto de todos los futuros profesores, pero sí que nos podemos plantear si este conjunto de futuros profesores (52 personas) se decanta claramente ("significativamente") por el "sí" o por el "no". En particular queremos saber si el porcentaje obtenido puede haberse obtenido al azar o se aleja tanto del $50 \%$ que es demasiado raro que ocurra al azar, y por tanto, refleja una tendencia más clara por el "sí".

\subsection{Análisis previo de los problemas}

Presentamos, los tres problemas de Lugares Geométricos planteados en el experimento de enseñanza a los futuros profesores de Secundaria. Se especifican distintas categorías: nivel, 
tipo de enunciado lugar geométrico, razonamiento visual-analítico, razonamiento instrumental, tipología de solución generada.

\subsubsection{Problema 1. Construcción de una elipse (método del jardinero)}

La elipse puede construirse como el lugar geométrico de los puntos cuya suma de distancias a dos puntos fijos es constante. Pista: Si se construye un segmento $\mathrm{AB}$, y un punto “móvil” E en el segmento, la suma de las distancias de los segmentos AE y EB es constante.

Nivel: Medio. ${ }^{4}$

Lugar geométrico: El enunciado del problema determina de una manera bastante directa los pasos a seguir en la construcción, salvo tal vez la idea de "puntos cuya suma de distancias a dos puntos fijos es constante”. Una vez aclarado este punto (se da una pista) la construcción es directa.

Razonamiento visual-analítico: La herramienta LugarGeométrico permite la visualización parcial del lugar, ya que sólo genera media elipse (es sencillo generar la otra media). Con EcuaciónLugar obtenemos la elipse entera, y además, podemos comprobar que se trata de una cónica mirando la ecuación. Con LocusGC obtenemos la misma solución.

Razonamiento instrumental: La construcción se realiza con elementos geométricos básicos (puntos, segmentos, rectas). La única dificultad es la utilización de un segmento auxiliar para determinar los radios de las circunferencias cuya suma es constante. La herramienta LugarGeométrico también permite una solución con otros elementos, típicamente deslizadores (esto no pasaba con versiones anteriores de GeoGebra). Sin embargo, el uso de deslizadores no es posible con EcuaciónLugar ni con LocusGC.

Como indicamos en la introducción en la construcción de algunos lugares geométricos clásicos, se dan algunas situaciones especiales que es necesario identificar. En este problema se presenta una situación en la que el lugar viene identificado por dos puntos móviles. Es lo que ocurre con la elipse: los dos puntos de intersección de la circunferencia describen la elipse completa. Si utilizamos la herramienta "LugarGeométrico" y queremos obtener toda la elipse,

\footnotetext{
${ }^{4}$ Hacemos notar que un problema puede ser sencillo de plantear, o tener un significado intuitivo claro, pero ser difícil de construir con GeoGebra, o puede tener un razonamiento matemático más complicado, pero venir descrito por un enunciado más sencillo. Entenderemos por nivel, el nivel de dificultad de la construcción instrumental.
} 
es necesario construir dos "lugares", cada uno correspondiente a un punto. Estas situaciones quedan solucionadas con la herramienta "EcuaciónLugar" que dibuja todo el lugar.

Tipología de la solución generada: En este caso, las 3 herramientas generan básicamente la misma solución, siendo más completa la generada por EcuaciónLugar.

\subsubsection{Problema 2. Construcción del Caracol de Pascal}

Este lugar se describe mediante el punto trazador T, dependiente del punto M. En este caso, $\mathrm{M}$ es un punto de la circunferencia con centro A que pasa por el punto B, y T está en la recta $\mathrm{BM}$ a una distancia fija de $\mathrm{M}$.

Nivel: Básico.

Lugar geométrico: El enunciado del problema permite la realización directa de la construcción.

Razonamiento visual-analítico: De nuevo, la herramienta LugarGeométrico genera únicamente una parte de la figura, el determinado por uno de los dos puntos de corte de la circunferencia y la recta, siendo difícil imaginar qué falta por construir, salvo que utilicemos de nuevo la herramienta con el otro punto, lo que nos permite una visión global del lugar (Fig.3). La herramienta EcuaciónLugar da todo el lugar, pero genera una componente "extraña" (una circunferencia) que proviene de un caso degenerado. Por tanto, la ecuación tampoco es correcta. Con LocusGC eliminamos este problema, ya que se quita la circunferencia extra, se tiene el lugar completo así como su ecuación precisa.

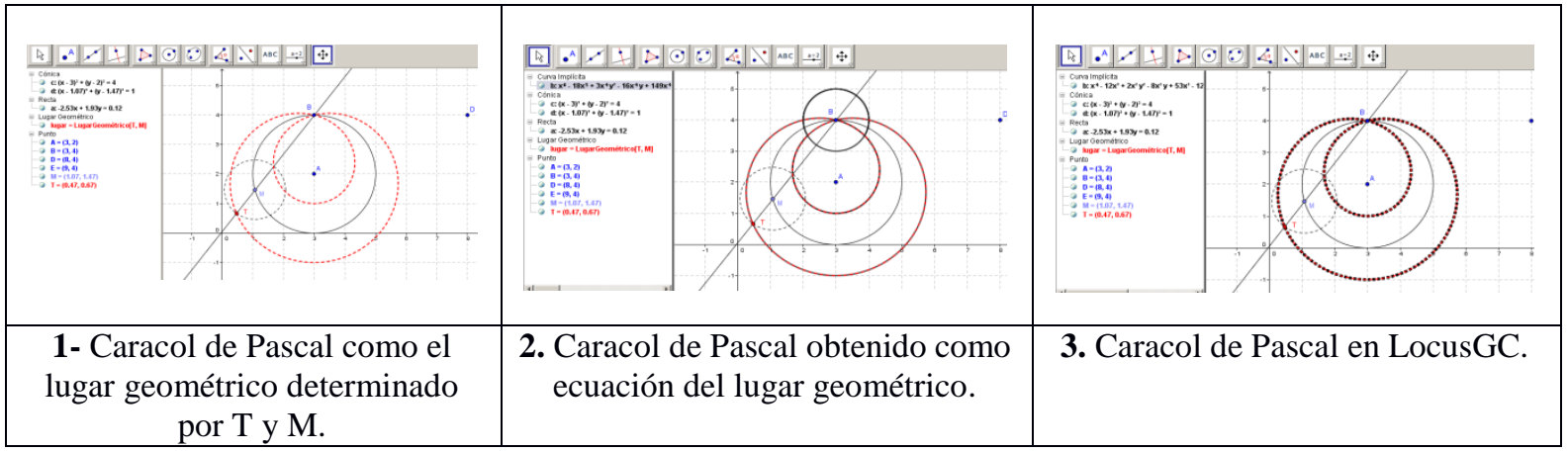

Figura 3 - Soluciones al problema 2 con cada una de las herramientas

Hacemos notar que un caso degenerado es un caso límite en el cual una clase de objeto cambia su naturaleza para transformarse, en una situación concreta, en un objeto de otra clase. Esto ocurre, por ejemplo, cuando en una construcción genérica se utilizan dos puntos distintos 
que, en algún momento, llegan a coincidir. Un caso degenerado, por tanto, tiene unas características especiales, que se apartan de las propiedades genéricas de la categoría más amplia y que, bajo una pequeña perturbación, podrían perderse.

Razonamiento instrumental: La construcción se realiza con elementos geométricos básicos (puntos, segmentos, rectas), válidos para las 3 herramientas.

Tipología de la solución generada: En este caso, las 3 herramientas estudiadas dan soluciones distintas. LugarGeométrico da una solución parcial, que es necesario completar con otro lugar geométrico. EcuaciónLugar da una solución completa, pero incluye elementos adicionales, correspondientes a casos degenerados que no se corresponden a la construcción general que queremos estudiar. En este problema 2, al de tener que realizar el dibujo en dos partes, aparece un caso degenerado cuando el punto $\mathrm{M}$ (móvil) se acerca al punto B (fijo). Esto crea una componente extraña (una circunferencia con centro B) que no pertenece al lugar geométrico clásico. La herramienta "EcuaciónLugar", con su maquinaria algebraica, dibuja esta circunferencia. Sin embargo, "LocusGC", utilizando un algoritmo de álgebra computacional, consigue un dibujo preciso sin las componentes extrañas, y por tanto da la solución más satisfactoria, tanto a nivel gráfico como a nivel algebraico (Fig. 4).

Además de esta resolución gráfica la Herramienta 3 (Fig.3) nos ofrece la ecuación de grado 4. Observamos que "EcuaciónLugar" da una ecuación de grado 6. Esta ecuación se obtiene en el panel "Vista Algebraica" de GeoGebra. Podemos manipular esta ecuación simplemente copiandola con ctrl+c.

RESULT: The sought locus (graphed in applet - dotted black) is the set:

$\mathbb{V}\left(x^{4}+2 x^{2} y^{2}+y^{4}-12 x^{3}-8 x^{2} y-12 x y^{2}-8 y^{3}+53 x^{2}+48 x y+33 y^{2}-102 x-64 y+56\right)$

Figura 4 - Ecuación de grado 4 del caracol de Pascal dada por "LocusGC”. "EcuaciónLugar” da una ecuación de grado 6.

\subsubsection{Problema 3. Construcción del lugar geométrico descrito por "El punto perdido"}

Este lugar se describe mediante el punto trazador T, dependiente del punto M. En este caso, sean A, B y $\mathrm{C}$ tres puntos fijos en el plano y $\mathrm{M}$ un punto en la recta $\mathrm{AB}$. El punto $\mathrm{T}$ es la intersección de la recta MC y la recta perpendicular a MC que pasa por B.

Nivel: Básico.

Lugar geométrico: El enunciado del problema permite la realización directa de la construcción. 
Razonamiento visual-analítico: La herramienta LugarGeométrico genera completamente la figura, una circunferencia. El mismo resultado genera la herramienta EcuaciónLugar, que da además la ecuación. Sin embargo, utilizando el dinamismo de GeoGebra observamos que al mover el punto que genera el lugar nos cuesta recorrer toda la circunferencia y además parece que hay un punto donde no es posible llegar (o que se llega "en el infinito"). Con LocusGC descubrimos que efectivamente hay un punto de la circunferencia que no está en el lugar, y nos indica cuál es.

Razonamiento instrumental: La construcción se realiza con elementos geométricos básicos (puntos, segmentos, rectas), válidos para las 3 herramientas.

Tipología de la solución generada: En este caso, las herramientas LugarGeométrico y EcuaciónLugar determinan la misma solución, una solución correcta desde el punto de vista algebraico pero incorrecta desde el punto de vista formal, ya que incorpora un punto que no está en el lugar. De eso nos podemos dar cuenta utilizando el dinamismo de la construcción. La herramienta "EcuaciónLugar" dibuja toda una circunferencia, cuando hay un punto que sólo se alcanza de forma asintótica. La herramienta "LocusGC" sí detecta este fenómeno y elimina el punto (no gráficamente, pero si a través de un mensaje).

Por último señalar que de cara al ejercicio del razonamiento visual-analítico y siguiendo el modelo de ETM que pone de manifiesto las transiciones entre génesis -figuralinstrumental-discursiva- consideramos muy interesante la información algebraica adicional que proporciona "EcuaciónLugar". Esto nos permite saltar de un registro visual-intuitivo a un registro matemático exacto. No solo hay dibujos, hay ecuaciones que se pueden calcular. Sin embargo, también consideramos importante la eliminación de información algebraica superflua. La eliminación de casos degenerados permite mostrar el "verdadero lugar" sin componentes que pueden tener un interés matemático, pero que desde un punto de vista educativo, no aportan nada. El ocultamiento de las componentes degeneradas permite exponer nítidamente la construcción matemática.

De distinta entidad son las situaciones especiales, como las mencionadas. Que el futuro profesor se haga consciente de las situaciones especiales y pueda detectarlas con la herramienta "LugarGeométrico" es "hacer visible lo invisible", trabajar las imágenes (los procesos de visualización) como "el poder de imaginar lo posible y lo imposible". La exploración gráfica de los conceptos matemáticos "no es una actividad cognitiva trivial: visualizar no es lo mismo que ver" (Hitt, 1997). Mostrar las diferentes representaciones del concepto permite ajustar para las situaciones de enseñanza el posible conflicto entre el 
concepto imagen en el individuo (ETM personal) y el concepto definición (ETM de referencia). El conocimiento detallado que el futuro profesor debe tener del objeto a representar es fundamental para revelar el contenido matemático profundo (no sólo gráfico) de los conceptos en estudio.

"LocusGC" no sólo proporciona dibujos precisos, libres de componentes extrañas, sino que además proporciona ecuaciones e identifica situaciones difíciles de localizar desde el punto de vista gráfico (como la existencia de puntos aislados) (Ver Fig. 3 y 4)

En el problema 3, la combinación de las 3 herramientas presentadas permite un estudio en profundidad, pero eliminando información espuria innecesaria (Fig. 5).

RESULT: The sought locus (graphed in applet - dotted black) is the set:

$$
\mathbb{V}\left(x^{2}+y^{2}-6 x-6 y+16\right) \backslash \mathbb{V}(y-4, x-4)
$$

Figura 5 - Ilustración: "LocusGC” identifica el "punto perdido" en el problema 3.

\section{Resultados}

Con objeto de dar respuesta a cómo se produce la evolución del concepto de lugar geométrico de este grupo de futuros profesores a través del uso de estas tres herramientas, esta sección se divide en dos partes. La primera, siguiendo el modelo ETM, se centra en la génesis instrumental a través de la descripción de las tipologías de soluciones de los participantes y en las dificultades observadas entre la génesis instrumental y la génesis figural visual en el uso de la herramienta. En la segunda parte, se caracteriza qué percepción tiene el futuro profesor de cara al uso profesional sobre algunas funcionalidades señaladas por el diseñador y que desde el modelo de ETM pueden ser consideradas funcionalidades didácticas para una mejor comprensión del concepto lugar geométrico.

\subsection{Cálculo de Lugares Geométricos: génesis instrumental y dificultades entre génesis}

En la Tabla 1 se recogen las frecuencias de tipologías de resolución matemática y procedimiento instrumental del grupo de estudio. En el problema 1 se destaca no sólo el número de casos no resueltos sino soluciones dadas para el lugar geométrico sólo mediante la utilización de "Traza" (Tabla 1). Es decir, se dibuja un punto y simplemente se observa el rastro que deja al ser desplazado. Esto da una solución intuitiva pero en absoluto precisa. El 
uso de la herramienta "LugarGeométrico" obliga a un proceso de modelización y conocimiento técnico de GeoGebra más elevado. Este proceso de modelización permite pasar de lo intuitivo a lo visualmente preciso, a costa de un esfuerzo técnico. Más de un $40 \%$ realizan una construcción no solo correcta, sino compatible con las herramientas utilizadas. Esto obliga a un mayor conocimiento técnico de GeoGebra y a un esfuerzo de modelización superior (el intuitivo deslizador no es válido para la herramienta "EcuaciónLugar" y obliga a otro tipo de construcciones auxiliares, como segmentos). Esto permite pasar de lo meramente visual a una compresión matemáticamente precisa de la construcción, que incluye una representación gráfica y las ecuaciones algebraicas.

Tabla 1 - Tipologías de solución y frecuencias $(\mathrm{N}=52)$.

\begin{tabular}{|c|c|c|c|c|c|c|c|}
\hline & \multicolumn{3}{|c|}{ Herramienta LugarGeométrico en GeoGebra } & \multicolumn{2}{c|}{$\begin{array}{c}\text { Herramienta "EcuaciónLugar" } \\
\text { en GeoGebra }\end{array}$} \\
\hline & LugarGeométrico & $\begin{array}{c}\text { LugarGeométrico } \\
\text { Deslizador }\end{array}$ & Traza & $\begin{array}{c}\text { No } \\
\text { resuelve }\end{array}$ & EcuaciónLugar & $\begin{array}{c}\text { Otra } \\
\text { Forma* }\end{array}$ & $\begin{array}{c}\text { No } \\
\text { resuelve }\end{array}$ \\
\hline Problema 1 & 16 & 21 & 8 & 7 & 19 & 15 & 18 \\
\hline Problema 2 & 23 & 14 & 11 & 4 & 22 & 8 & 22 \\
\hline Problema 3 & 34 & 1 & 13 & 4 & 30 & 6 & 16 \\
\hline
\end{tabular}

* Otra forma = otra manera de encontrar la solución, encontrando las ecuaciones de maneras alternativas.

Como se indicó en el análisis previo del problema 1 este requiere una modelización anterior al uso de la herramienta LugarGeometrico. Para la ejecución de esta modelización los conocimientos previos generaron ciertas dificultades relativas a las génesis del trabajo geométrico. Algunos estudiantes, al leer "método del jardinero" ya no leían más del enunciado y pasaban a una construcción que recordaban haber estudiado anteriormente. El manejo de dos distancias cuya suma es constante (sencillo de hacer utilizando un segmento auxiliar) constituyó para ellos un bloqueo en la resolución. 
Tabla 2 - Tipologías de dificultades asociadas a las génesis expresadas con la herramienta 1 LugarGeometrico.

\begin{tabular}{|c|c|c|}
\hline $\begin{array}{l}\text { D1-Geo. Dif. Comprensión e } \\
\text { Interpretación problema }\end{array}$ & & $11(21.15 \%)$ \\
\hline $\begin{array}{l}\text { D2-Geo. Dif. En relación a la } \\
\text { génesis figural visual }\end{array}$ & & $4(7.7 \%)$ \\
\hline \multirow{2}{*}{$\begin{array}{l}\text { D3-Geo.Dif. asociadas con la } \\
\text { génesis instrumental }\end{array}$} & $\begin{array}{l}\text { D31-Geo. Dificultades asociadas con los comandos del } \\
\text { software y el significado matemático }\end{array}$ & $13(25 \%)$ \\
\hline & $\begin{array}{l}\text { D32-Geo. Dificultades sobre dependencia de objetos en } \\
\text { geometría dinámica }\end{array}$ & $10(19.2 \%)$ \\
\hline \multirow{4}{*}{$\begin{array}{l}\text { D4-Geo. Bloqueo en el control } \\
\text { global de las diferentes génesis de } \\
\text { trabajo geométrico }\end{array}$} & $\begin{array}{l}\text { D41-Geo. Bloqueo del paso del razonamiento } \\
\text { discursivo al instrumental }\end{array}$ & $10(19.2 \%)$ \\
\hline & $\begin{array}{c}\text { D42-Geo. Bloqueo del paso del razonamiento visual al } \\
\text { analítico-algebraico }\end{array}$ & $6(11.53 \%)$ \\
\hline & $\begin{array}{c}\text { D43-Geo. Bloqueo del paso del razonamiento visual al } \\
\text { razonamiento instrumental }\end{array}$ & $7(13.46 \%)$ \\
\hline & D44-Geo. Bloqueo en el uso de LOCUS & $13(25 \%)$ \\
\hline
\end{tabular}

En la resolución con la herramienta 1 (LugarGeométrico de GeoGebra) se constata que el $25 \%$ de los estudiantes señala dificultades en la utilización del comando (Tabla 2), mostrando dificultades asociadas con la génesis instrumental en un $44.2 \%$ y produciéndose bloqueos del paso del razonamiento discursivo al instrumental en un 19.2\%.

Algunas de sus justificaciones representativas son:

El primer problema resultó el más complicado al no tener una referencia clara de qué forma definir la dependencia para el lugar geométrico" (JU., Cuestionario1)

"En el caso del caracol de Pascal (Problema 2), lo dificil fue hacer que los puntos $S$ y $T$ estuviesen a una distancia constante de M. Primero intenté poner un punto en la recta que pasa por $M$, pero al mover el punto $M$ a lo largo de la circunferencia vi que la distancia no se mantenía constante, así que decidí meterle el punto al GeoGebra mediante coordenadas, aunque también podía haber hecho la intersección de una circunferencia centrada en $M$ con la recta que pasa por M. (JV., Cuestionario 1)

El análisis estadístico jerarquizado implicativo de las resoluciones de los 52 estudiantes de los tres problemas mediante las variables tipología de resolución con las herramientas Herramienta "LugarGeométrico", "EcuaciónLugar" en GeoGebra y "LocusGC" y tipología de dificultad indica una relación causal con un índice de fiabilidad de al menos un $80 \%$ entre las siguientes variables:

- D4Geo (Bloqueo en el control global de las diferentes génesis de trabajo geométrico Herramienta "LugarGeométrico") - (.80) $\rightarrow$ D32Geo. Dificultades sobre dependencia de objetos en geometría dinámica- $(.80) \rightarrow R P 3-E C$ (resolución del problema 3 mediante "EcuaciónLugar").

- D43-Geo Bloqueo en el paso del razonamiento visual al razonamiento instrumental $-(.80) \rightarrow$ D3Geo. Dificultades asociadas con la génesis 
instrumental-(.90) $\rightarrow R P 1-L G$ (Resolución del problema 1 mediante la herramienta 1 Lugar-Geométrico).

Y respecto a la herramienta "LocusGC", las implicaciones más significativas están (con índices de fiabilidad del 90\%) entre $D 42 W$ - Bloqueo en el paso del razonamiento visual analítico al algebraico y las D3Geo (Dificultades asociadas a la génesis instrumental) y la dependencia de estas dificultades con el modo de resolución con LugarGeométrico en GeoGebra en los problemas 1 y 3 . Este resultado pone de manifiesto que aquellos sujetos que han sido capaces de resolver correctamente los problemas 1 y 3 mediante la herramienta "Ecuación Lugar" en GeoGebra y la herramienta "LocusGC" han aportado más información y son conscientes de dificultades asociadas con la génesis instrumental y las dificultades en la transición entre génesis semiótico-figural e instrumental y discursiva.

En síntesis, sujetos que informan más conscientemente sobre este tipo de dificultades son aquellos que han resuelto los problemas por "LugarGeométrico" o por "LugarGeométrico con deslizador". Este nivel de consciencia puede implicar que su espacio de trabajo personal es más preciso matemáticamente. Además, son estos mismos sujetos los que avanzan en la resolución de los problemas utilizando las otras herramientas y son más capaces de caracterizar las funcionalidades didácticas como profesionales (sec.4.2).

\subsection{Uso profesional como futuros profesores: funcionamiento semiótico-figural, instrumental y discursivo}

Tal como se indicó en la sección 3 (análisis previos de los problemas y metodología) se explora si se produce alguna relación en su concepción de lugar geométrico entre la génesis instrumental y la integración de las herramientas respecto a modalidades de empleo y funcionalidades en clave en aprendizaje matemático. Los resultados ponen de manifiesto 1) conexiones entre la valoración de las herramientas y funcionamiento matemático (aspectos epistemológico y formas de resolución de los problemas); y 2) en relación a la transición entre génesis figural y génesis discursiva, ésta se muestra inestable manifestándose en la no identificación de situaciones especiales y casos degenerados y en el establecimiento de sinergia entre el registro gráfico y el registro analítico-algebraico.

\subsubsection{Valoración de las herramientas y funcionamiento matemático}


En la valoración de las herramientas se tuvieron en cuenta distintos criterios de calidad de acuerdo con la finalidad epistemológica planteada por el diseñador y el punto de vista de usos profesionales en Secundaria: el primer nivel de evaluación para cada solución/imagen esperada, exactitud-precisión matemática y satisfacción con el resultado matemático (Tabla 3).

Tabla 3 - Criterios de calidad para la herramienta (porcentajes y dominios).

\begin{tabular}{|c|c|c|c|}
\hline & $\begin{array}{c}\text { Herramienta } \\
\text { "LugarGeométrico" en } \\
\text { GeoGebra }\end{array}$ & $\begin{array}{c}\text { Herramienta } \\
\text { "EcuaciónLugar" en } \\
\text { GeoGebra }\end{array}$ & $\begin{array}{c}\text { Herramienta } \\
\text { "LocusGC" }\end{array}$ \\
\hline $\begin{array}{c}\text { Valoración de lo } \\
\text { esperado }\end{array}$ & $88.5 \%(46 \mathrm{p})$. & $55.7 \%(29 \mathrm{p})$. & $51.9 \%(27 \mathrm{p})$ \\
\hline Valoración de exactitud & $71.2 \%(37 \mathrm{p})$ & $59.6 \%(31 \mathrm{p})$ & $59.6 \%(31 \mathrm{p})$ \\
\hline $\begin{array}{c}\text { Valoración de } \\
\text { Satisfacción }\end{array}$ & $86.53 \%(45 \mathrm{p})$ & $57.70 \%)(30 \mathrm{p})$ & $57.70 \%(30 \mathrm{p})$ \\
\hline
\end{tabular}

El grupo considera con más alta valoración la herramienta 1 "LugarGeométrico" en GeoGebra. Las razones que alegan están más relacionadas con el espacio de trabajo "idóneo" -producto de la transposición que intenta hacer de un conocimiento para enseñarlo en el aula de Secundaria- que con su propio estudio como "matemático". Entre sus argumentos destacamos:

a) Herramienta 1 "LugarGeométrico", una herramienta más apropiada para la comprensión y profundización en las propiedades geométricas, sirve más para explorar y trabajar el razonamiento geométrico y visual con los alumnos de Secundaria.

b) Herramienta 1 "LugarGeométrico", una herramienta más intuitiva.

c) La herramienta 1 "LugarGeométrico" versus herramienta 2 "EcuaciónLugar" porque la primera coincide con la imagen mental que puede formase el alumno de Secundaria.

También, se pone de manifiesto una serie de conexiones entre la valoración de las herramientas, las formas de resolución de los problemas y la percepción e imagen mental inicial de los lugares geométricos en los tres problemas. El análisis estadístico jerarquizado implicativo de las resoluciones de los 52 futuros profesores a través del grafo implicativo indica una relación causal con un índice de fiabilidad de al menos un $90 \%$ que los resolutores que perciben (imagen previa de la solución) el Lugar Geométrico en el Problema 2 y en el Problema 1 son los que realmente han realizado la resolución mediante el comando "LugarGeométrico". 
Además, las clases generadas en árbol de similitudes y del árbol cohesitivo del análisis estadístico jerarquizado implicativo nos llevan a establecer una serie de relaciones que se pueden precisar del modo siguiente:

- Existe un grupo de futuros profesores que resuelven con traza todos los problemas. Dan por válido esta aproximación gráfica y no buscan otras soluciones más precisas geométrica y algebraicamente.

- Los que resuelven los tres problemas con herramienta 2 "EcuaciónLugar" dan una valoración positiva de la herramienta 3 "LocusGC" (índice de cohesión de 0.923) y son capaces de hacer una interpretación matemáticamente correcta sobre la solución identificando puntos degenerados y diferencias con la herramienta 2. (índice de cohesión de 0.822).

\subsubsection{Transición entre génesis figural y génesis discursiva}

Como se mostró en la sec. 2, la visión experta del desarrollador destacaba como funcionalidad de la herramienta "LocusGC" la solvencia de la problemática de la continuidad y la sinergia entre el registro gráfico y el registro analítico-algebraico a través de la producción formal y exacta de resultados. Los datos ponen de manifiesto que entre los futuros profesores, muchos de ellos no aprecian la problemática de la continuidad en el paradigma de SGD, no identificando estos casos degenerados.

MA.: En el problema 3, me fije en el título y luego moví para ver que había. Respecto al enunciado este es el problema más fácil, pero luego fue el que menos entendí. Lo del punto ciego, cuando tracé el lugar geométrico, que ya lo tenía, que veía que cumplía las propiedades que me había dicho, me preguntaba qué querrá decir esto del punto ciego... Le di vueltas. Traté de ver intuitivamente que era. Busqué la visualización de ese punto ciego moviendo el punto $T$ ver que significaba lo del punto ciego. El lugar geométrico era una circunferencia, ¿pero esto qué quiere decir de punto ciego? Si es verdad que había tres puntos $A, B$ y $C$, un punto $M$ que pertenece a una de las dos rectas y tú al moverte es verdad que según movías $M$, se creaba una posición que parecía oculto, por $M$ o por otro punto respecto a A y B (ahora no sé exactamente). Me quedó la duda si el nombre del problema era por eso o no. No le vi todo el sentido

Investigador 1: ¿Viste el mensaje que te sale al respecto en la herramienta "LocusGC”? MA.: No no vi nada, no me fijé. (el investigador le muestra con el ordenador el mensaje) MA.: ah!! es ese punto no pertenece al lugar geométrico, es todo menos ese punto (MA., entrevista, video 28, 10-13:10)

Entre aquellos sujetos que sí son conscientes se plantea la discusión sobre qué herramienta es más pertinente en Secundaria y si en Secundaria es beneficioso mostrar de forma ostensible los puntos degenerados a un estudiante: 
1:42 Investigador 2: ¿Y con lo del punto perdido? El tercero, ¿Lo viste? ¿Lo identificaste? ¿Cómo fue?

1:47 JU: El punto ciego, sí, el punto ciego se identifica de la misma manera que con la segunda práctica del lazo. Que es... tú vas moviéndolo de forma manual sobre la recta el punto deslizante, entonces lo que te das cuenta es que va describiendo el... el punto que te describe el lugar geométrico, va recorriendo la circunferencia, pero realmente el punto diametralmente opuesto al de la recta, al tangente a la recta, no lo alcanzas nunca, se alcanzaría en tiempo infinito y vuelve a hacer lo mismo, ahí lo único es que no lo aproxima, no es que lo aproxime y te ponga el punto sino que... te cubre todo, menos un punto. Entonces, visualmente es la circunferencia, pero realmente tienes que distinguir que ese punto tal y como has definido el lugar geométrico, no pertenece a la figura (JU., entrevista, video $32,1: 42-2: 34)$.

Respecto a estos aspectos ostensibles de continuidad, en el grupo de futuros profesores las herramientas "LocusGC" y "EcuaciónLugar" son percibidas como herramientas que pueden propiciar la sinergia entre una intuición gráfica del concepto y una intuición analíticaalgebraica contribuyendo a la articulación entre génesis:

\begin{abstract}
Por ejemplo, en el primero una ventaja que le encuentro es el utilizar por ejemplo la herramienta "EcuaciónLugar", pues para un alumno la construcción, ver cómo se construye una elipse mediante una ecuación-lugar... Me parece bastante relevante que como profesor intentemos que el alumno también pueda ver cómo se construye gráficamente y qué interpretación tiene la ecuación, o sea, qué significa esa ecuación. Pues en la elipse era tomar los dos focos fijos e ir tomando circunferencias, una iba aumentando y otra disminuyendo y los puntos de corte eran los que te iban definiendo la elipse, otra vez en dos partes, pero bueno. $Y$ es el método del jardinero, que en dibujo técnico es como se estudia y es cómo surgió el dibujo de la elipse. Un alumno no lo sabe, entonces como profesores me parece bastante didáctico mostrarles cómo hacerlo. También por ejemplo, una vez pasamos a la ecuación-lugar con la elipse, el ver que la elipse... la ecuación de la elipse no siempre tiene los coeficientes bonitos, por decirlo así, entre comillas. No tiene por qué ser agradables, si no que nosotros damos una... dibujamos una elipse y puede tener unos coeficientes que sean muy grandes, fracciones, decimales... algo que el alumno normalmente no... no se plantea. En un examen por ejemplo, lo más común es ponerles la ecuación con, pues con coeficientes enteros... de máximo dos cifras... pero luego realmente cuando ellos dibujan, como no les das una limitación en cuanto a dónde colocar los focos, el que vean que no siempre va a ser una ecuación bonita, también les va a sorprender a ellos y les va a ayudar a comprender que no todo es simple, o sea, no es tan simple (Entrevista JU., Video 32, 3:06- 6:53).
\end{abstract}

La insistencia al comparar las Herramientas "EcuaciónLugar" y "LocusGC” acentúa la necesidad del conocimiento del profesor respecto al significado de las discontinuidades que actúan en la construcción del lugar geométrico y cómo quedan expresadas algebraicamente:

Para el segundo problema. Y sobre todo también, como profesor, futuro profesor, me parece curioso el que comparen los alumnos si les ha parecido la incongruencia de la circunferencia en la segunda parte, cómo desaparece utilizando el programa de Internet y que comparen sobre todo, porque gráficamente se ve, pero que comparen también las ecuaciones. Y que vean que el grado de la ecuación ha disminuido. Y que intenten ver por qué ocurre eso, por qué surge eso. El profesor lo puede saber, porque desaparece una circunferencia que han añadido, que tiene grado dos, con lo cual al introducirla en la ecuación del lugar geométrico te aumenta en dos la ecuación de lugar total. Entonces, ver ese cambio... ha depurado realmente el programa de Internet, ha depurado los problemas que surgían de la 
discontinuidad. Entonces me parecía bastante útil en ese sentido (Entrevista JU., Video 32,8.47-9:46)

La percepción de los futuros profesores sobre la competencia de las herramientas desde el punto de vista de tipos de funcionamiento semiótico-figural, instrumental y discursivo pone de manifiesto algunos aportes útiles para el educador matemático y para el desarrollo de la herramienta en un ETM más efectivo. En las herramientas bajo el paradigma de los SGD la resolución intuitiva está cristalizada con frecuencia en una concepción cerrada en la incompletitud y la vaguedad de información está enmascarada mediante mecanismos especiales que producen un sentimiento de inmediatez, coherencia y confianza. Inmediatez en el sentido de evidencia intrínseca y la coherencia y la confianza - en el sentido de la certeza.

\section{Conclusiones}

El análisis del ETM personal indica que en una proporción amplia de futuros profesores se produce una deficiencia heurística ("esa incapacidad de ir más allá de lo que se ve en un primer vistazo") en la interpretación geométrica de las visualizaciones, en este caso en la comprensión de Lugar Geométrico desde el punto de vista funcional. Los datos muestran que la función heurística en la génesis figural exige de la deconstrucción visual de las formas perceptivas elementales que se imponen a golpe de vista. Un número significativo de sujetos sólo se quedan en la aprehensión local (uso de la traza), y no son capaces de llegar a lo global (uso del comando LugarGeometrico). Sin embargo, aquellos sujetos que resuelven los tres problemas con herramienta 2 "EcuaciónLugar" dan una valoración positiva de la herramienta prototipo "LocusGC" cuyas funcionalidades contribuyen a una interpretación matemáticamente correcta de la solución, identificando puntos degenerados y la problemática de la continuidad en SGD. Este resultado conlleva una llamada de atención para el uso profesional como educador, poniéndose de manifiesto que cuando se trabaja con geometría dinámica la aprehensión perceptual, que sirve para ver las figuras y las visualizaciones icónica no siempre conduce a la aprehensión operativa (DUVAL, 2005).

La caracterización realizada por los futuros profesores, que cualifica a las herramientas respecto a modalidad de funcionamiento semiótico-figural, instrumental y discursivo y sus sinergias en el aprendizaje, pone de manifiesto que estas dependen del funcionamiento matemático de los sujetos (aspectos epistemológicos y formas de resolución de los problemas); y de los bloqueos o dificultades en relación a la transición entre génesis figural y génesis 
discursiva, por ejemplo, en la no identificación de situaciones especiales y casos degenerados. Resultado que confirma que el paso del mundo numérico al mundo algebraico no está exento de problemas. La herramienta "LocusGC" pretende cubrir esta problemática, y así es apreciado por algunos estudiantes.

Todo esto indica que en la formación de futuros profesores no se debe descuidar el estudio de herramientas educativas cómo GeoGebra, no únicamente desde un punto de vista didáctico, sino que es necesaria una formación técnica para desarrollar toda la potencialidad de la herramienta. Además, tampoco se debe olvidar que, para analizar todo tipo de situaciones, es necesario tener unos conocimientos matemáticos que es necesario reforzar.

Por último, indicar que las funcionalidades didácticas proporcionadas desde el diseñador han sido estudiadas desde el modelo ETM. Desde este modelo el desarrollo cognitivo del individuo se articula a través de tres génesis fundamentales visual-semiótica, instrumental, discursiva. Ha sido de utilidad para el estudio el rol asignado a la herramienta como aspecto esencial en la conexión entre el nivel epistemológico y el nivel cognitivo y también en la exploración de la evolución del concepto de Lugar Geométrico a través de las distintas herramientas. Ahora que la herramienta prototipo LocusGC ha sido integrada en el SGD de GeoGebra, el uso de este modelo puede potenciar otras experimentaciones similares realizadas en un contexto de formación del profesorado y con el planteamiento de situaciones de homología sobre resultados aberrantes (inexactos, imprecisos) debidos a discontinuidades o errores numéricos en un contexto de aprendizaje matemático con tecnología.

\section{Agradecimientos}

Este trabajo ha sido parcialmente financiado por el Ministerio de Economía y Competitivida español y por la European Regional Development Fund (ERDF), bajo el proyecto MTM201454141-P. También, mostramos nuestro agradecimiento a los revisores del artículo sus críticas y sugerencias ha dado impulso a este trabajo.

\section{Referencias}

ABÁNADES, M.; BOTANA, F.; ESCRIBANO, J.; GÓMEZ-CHACÓN, I. M. Distintas herramientas para la enseñanza/aprendizaje del concepto de lugar geométrico. In: GÓMEZ-CHACÓN, I. M. et. al (Ed.). Mathematical Working Space, Proceedings Fourth ETM Symposium. Madrid: Publicaciones del Instituto de Matemática Interdisciplinar, Universidad Complutense de Madrid. 2015. p. 261-282. 
ARZARELLO, F., OLIVERO, F., PAOLA, D., ROBUTTI, O. A cognitive analysis of dragging practises in Cabri environments. ZDM- Zentralblatt für Didaktik der Mathematik, Berlin, v.34, n.3, p. 66-72, jun. 2002.

BALACHEFF, N.; KAPUT, J. Computer-based learning environment in mathematics. In: A. J. BISHOP, A. J. et al. (Ed.). International handbook of mathematical education. Dordrecht: Kluwer Academic Publishers, 1996. p. 469-501.

BOTANA, F. Interactive versus symbolic approaches to plane loci generation in dynamic geometry environments. Lectures Notes in Computer Science, Berlin, v. 2330, p. 211-218. 2002.

BOTANA, F. Bringing more intelligence to dynamic geometry by using symbolic computation. In: SHANGZHI L.; DONGMING W.; JING-ZHONG Z. (Ed.). Symbolic Computation and Education. Singapur: World Scientific, 2007. p. 136-150.

BOTANA, F.; ABÁNADES, M. A. Automatic deduction in (dynamic) geometry: loci computation, Computational Geometry: theory and applications, Amsterdam, v.47, n.1, p. 75-89, ene. 2014.

CERULLI, M.; PEDEMONTE, B.; ROBOTTI, E.. An integrated perspective to approach technology in mathematics education. In: BOSCH, M. (Ed.). Proceedings of CERME 4 Sant Feliu de Guíxols: IQS Fundemi Business Institute, 2006. p. 1389-1399.

COUTURIER, R. CHIC: utilisation et fonctionnalités. In: GRAS, R.; ORÚS, P.; PINAUD, B.; GREGORI, P. (Ed.). Nouveaux apports théoriques à l'analyse statistique implicative et applications Castellón: Universitat Jaume I, 2007. p. 41-49.

DUVAL, R. Les conditions cognitives de l'apprentissage de la géométrie: développement de la visualization, différenciation des raisonnements et coordination de leurs fontionnements. Annales de Didactique et de Sciences Cognitives, Strasbourg, v.10, p. 5-53. 2005.

GÓMEZ-CHACÓN, I. M.; ESCRIBANO, J. Geometric locus activities in a dynamic geometry system. Non-iconic visualization and instrumental genesis. RELIME, Revista latinoamericana de investigación en matemática, México D.F .,v.17, n.4-II, p.361-383, dic. 2014.

GÓMEZ-CHACÓN, I. M.; KUZNIAK, A. Spaces for geometrical work: figural, instrumental and discursive geneses of reasoning in a technological environment, International Journal of Science and Mathematics Education, Dordrecht, v.13, n. 1, p.201-226, feb. 2015.

GONSETH, F. La géométrie et le problème de l'espace. Lausanne: Éditions du Griffon, 1945.

GRAS, R.; PETER, P.; BRIAND, H.; PHILIPPE, J. Implicative statistical analysis. In: HAYASHI, C. et al. (Ed.). Data Science, Classification, and Related MethodsNew York: Springer-Verlag, 1998. p. 412-419.

HITT, F. Researching a problem of convergence with Mathematica: history and visualisation of a mathematical idea. International Journal of Mathematical Education in Science and Technology, Abingdon, v. 28, n. 5, p. 697-706. 1997.

JACKIW, N. Attention to detail; broadening our design language.In: HOYLES, C; LAGRANGE, J. B. (Ed.). Mathematics Education and Technology-Rethinking the Terrain New York: Springer. 2010. p. 431-433. 
JAHN, A. P. "Locus" and "Trace" in Cabri-géomètre: relationships between geometric and functional aspects in a study of transformations. ZDM- Zentralblatt für Didaktik der Mathematik, Berlin, v. 34, n. 3, p. 78-84, jun. 2002.

KUZNIAK, A. L'espace de travail mathématique et ses genèses. Annales de Didactique et de Sciences Cognitives, Starsbourg, v. 16, p. 9-24. 2011.

LABORDE, C. Integration of technology in the design of geometry tasks with Cabri-geometry. International Journal of Computers for Mathematical Learning, Amsterdam, v. 6, n. 3, p. 283317, ene. 2002.

LAGRANGE, J-B. (Dir.). Les technologies numériques pour l'enseignement - Usages, dispositifs et genèses. Toulouse: Octares, 2013.

MONTES, A.; WIBMER, M. Gröbner bases for polynomial systems with parameters. Journal of Symbolic Computation, London, v. 45, n. 12, p. 1391-1425, dic. 2010.

SUTHERLAND, I. E. Sketchpad: a man-machine graphical communication system. In: WOLFE, R. (Ed.) Seminal graphics: pioneering efforts that shaped the field New York, ACM Press. 1998. p. 391-408.

Submetido em Julho de 2015. Aprovado em Setembro de 2015. 


\section{Anexos}

\section{Anexo 1: Protocolo de resolución}

Para cada uno de los problemas realiza un protocolo detallado de tu proceso de resolución del mismo. Puedes utilizar el formato que viene a continuación. Ayúdate de imágenes del GeoGrebra para hacer la descripción. Además de hacer el protocolo deberás adjuntar el archivo GeoGebra.

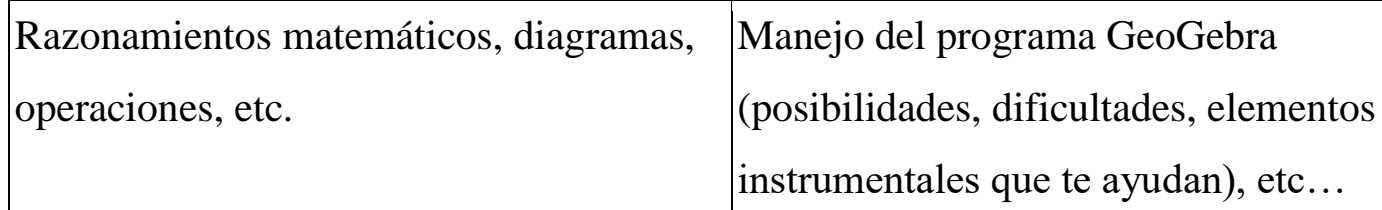

Anexo 2: Ejemplo de preguntas de los cuestionarios, en este caso el Cuestionario 1 online: Herramienta, competencia y preferencias

Una vez hallado el lugar geométrico mediante la herramienta LUGAR GEOMETRICO responde a las siguientes preguntas.

1. ¿La gráfica que obtienes representa todo el lugar geométrico? ¿Por qué?

2. Valora a continuación de 1 a 5 si la respuesta obtenida se corresponde con lo esperado.

3. Valora a continuación de 1 a 5 si la respuesta obtenida es matemáticamente exacta.

4. Valora a continuación de 1 a 5 si la respuesta obtenida es satisfactoria.

5. Describe cuales han sido tus bloqueos y tus emociones al trabajar el problema.

Señala si tus dificultades coinciden con algunas estas.

D1: Dificultades en la comprensión e interpretación del problema, fase inicial de la resolución del problema.

D2: Dificultades en relación a la génesis visual-figural (visualización)

D3: Dificultades asociadas con la génesis instrumental

D31 Dificultades asociadas con los comandos del software y el significado matemático

D32 Dificultades sobre dependencia de objetos en geometría dinámica

D4: Bloqueo en el control global de las diferentes génesis del trabajo geométrico

D41 Bloqueo del paso del razonamiento discursivo al instrumental

D42 Bloqueo del paso del razonamiento visual al analítico-algebraico.

D43 Bloqueo del paso del razonamiento visual al razonamiento instrumental

D44 Bloqueo en el uso de LUGAR GEOMETRICO

Indica qué estrategias y recursos para superar tus bloqueos en el problema has utilizado. 